\title{
Öber die Herkunft der schwefelhaltigen Stoffwechselprodukte im tierischen Organismus.
}

\author{
Mitteilung I. \\ von
}

J. Wohlgemuth.

(Aus dem chemischen Laboratorium des pathologischen Instituts der Universität Berlin.)

(Der Redaktion zugegangen am 7. Oktober 1903.)

Während man bis vor kurzem sich noch garnicht klar war über die Quelle der schwefelhaltigen Stoffwechselprodukte, speziell des Taurins in der Galle, trat durch Mörners ${ }^{1}$ ) bedeutungsvolle Entdeckung, daß bei weitem die Hauptmenge des im Eiweiß enthaltenen Schwefels sich in Form von Cystin abspalten läßt, wenigstens die theoretische Möglichkeit von dem Zusammenhang der Taurinbildung mit der Cystingruppe in den Vordergrund. Allein erst durch die Arbeiten von C. Neuberg ${ }^{2}$ ) und E. Friedmann, ${ }^{3}$ ) durch die auch die wahre Konstitution des Cystins erkannt wurde, ist das experimentelle Band zwischen der schwefelhaltigen Eiweißgruppe und dem Gallenbestandteil geschlungen.

Neuberg zeigte, daß man durch direkte Oxydation des Cysteins mittels Salpetersäure zur Isäthionsäure gelangt. Letztere steht zum Taurin im Verhältnis von $0 x y-z u$ Aminosäure und kann künstlich leicht aus ihm gewonnen werden, wie beistehende Formel lehrt:

$$
\begin{aligned}
& \mathrm{CH}_{2} \cdot \mathrm{NH}_{2} \\
& \mathrm{CH}_{2} \cdot \mathrm{SO}_{3} \mathrm{H}
\end{aligned}
$$

Taurin

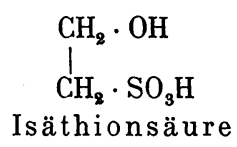

1) K. A. H. Mörner, Diese Zeitschr., Bd. XXVIII, S. 595; Verh. des medizin. Kongr. in Paris 1900, Section de physiologie, physique et chimie biologique, Bd. 8, S. 15.

2) C. Neuberg, Berichte d. dtsch. chem. Gesellsch. Bd. XXXV, S. 3161. 1902.

3) E. Friedmann, Hofmeisters Beiträge, Bd. 2, S. 433. 1902. 
Auf Umwegen gelangte Friedmann vom Cystin zum Taurin, indem er das Cystein zur Aminosulfopropionsäure oxydierte und diese durch Abspaltung von $\mathrm{CO}_{2}$ in Taurin überführte.

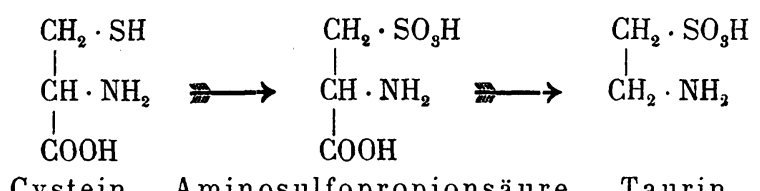

Cystein Aminosulfopropionsäure Taurin

An diesen Verhältnissen wird nichts geändert durch die neuen Befunde von C. Neuberg und P. Mayer, ${ }^{1}$ ) daß man zwischen zwei Cysteinformen - der $\alpha$-Thio- $\beta$-aminopropionsäure, die sich in Cystinsteinen findet, und der $\alpha$-Amino$\beta$-thiopropionsäure, die im Körpereiweiß enthalten ist zu unterscheiden hat. Im Gegenteil ist das neu aufgefundene. Thioisoserin besonders zum Übergang in Taurin geeignet.

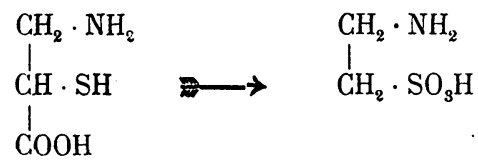

Hierfür bedarf es lediglich einer Oxydation und der $\mathrm{CO}_{2}-\mathrm{Ab}$ spaltung, zweier physiologischer Prozesse, von denen der erste aus zahlreichen Fällen seit langem bekannt ist, während für den letzteren grade in jüngster Zeit eine Reihe von Beispielen vorliegt, so die Bildung von p-Kresol aus Oxyphenylessigsäure nach Baumann, $\left.{ }^{2}\right)$ von Cadaverin aus Lysin und von Putrescin aus Ornithin nach Ellinger, ${ }^{2}$ ) von l-Xylose aus d-Glukuronsäure nach E. Salkowski und C. Neuberg. ${ }^{2}$ )

Die Frage nach der Herkunft der schwefelhaltigen Stoffwechselpropukte beschäftigt schon seit langem die physiologischen Chemiker. Vor mehr als 30 Jahren bereits stellte E. Salkowski ${ }^{3}$ ) mit Taurin Versuche an Kaninchen, Hunden und an Menschen an und konnte die bemerkenswerte Tatsache

1) C. Neuberg u. P. Mayer, Über Cystein II, vorgetragen in d. dtsch. chem. Gesellsch. zu Berlin, 25. Mai 1903.

$\left.{ }^{2}\right)$ Literaturangaben s. Diese Zeitschr., Bd. XXXVI, S. 261.

3) E. Salkowski, Virchows Archiv, Bd. LVIII, S. 460. 1873. 
feststellen, daß bei Kaninchen nach Dosen von 3-6 g Taurin per os im Harn eine vermehrte Ausscheidung des oxydierten, mehr aber noch des nichtoxydierten Schwefels auftritt und gleichzeitig damit eine beträchtliche Menge von unterschwefligsaurem Natron. Anders gestaltete sich das Resultat beim Hunde und beim Menschen; bei beiden konnte selbst nach Verabreichung größerer Mengen Taurin (bis zu $10 \mathrm{~g}$ ) keine gesteigerte Schwefelsäureausfuhr und auch keine unterschwetlige Säure im Harn konstatiert werden. Desgleichen führte E. Salkowski Fütterungsversuche mit Isäthionsäure ${ }^{1}$ ) aus und stellte im Harn der Tiere einen vermehrten Gehalt an Schwefel und die Anwesenheit unterschwefliger Säure fest. Wenn auch später Heffter ${ }^{2}$ ) zu abweichenden Resultaten gekommen war, so kann Salkow skis ${ }^{3}$ ) nochmalige Nachprüfung der Versuche als völlig beweisend für seine zuerst gemachten Befunde angesehen werden. Über die Zwischenprodukte zwischen dem Schwefel und Schwefelsäure des Harns eröffneten einen überraschenden Einblick die Untersuchungen von Baumann ${ }^{4}$ ) und von Jaffé. ${ }^{5}$ ) Beide fanden - unabhängig von einander - , daß bei Hunden nach Verfütterung von Brom-, Chlor- oder Jodbenzol im Harn Merkaptursäuren auftreten. Goldmann ${ }^{6}$ ) setzte die Versuche von Baumann fort und konstatierte sowohl bei gesteigerter Chlorbenzolfütterung als auch bei Darreichung von Cystin, daß das Verhältnis des oxydierten zum nichtoxydierten Schwefel im Harn fast konstant $2: 1$ ist. Da nun das verfütterte Cystin aus Cystinsteinen stammte, dieses aber vom Eiweißcystin wesentlich verschieden ist, schien es zweckmäßig, Versuche mit Eiweißcystin an Tieren anzustellen und dessen Schicksal im tierischen Organismus zu verfolgen.

Das zur Verfütterung verwandte Cystin stammte aus

1) E. Salkowski, Virchows Archiv, Bd. 66, S. 315.

2) Heffter, Pflügers Archiv, Bd. 38, S. 476.

3) E. Salkowski, Pflügers Archiv, Bd. 39, S. 209.

4) Baumann u. Preuße, Ber. d. dtsch. chem. Gesellschft., Bd. 12, S. 806; Diese Zeitschr., Bd. V, S. 309.

5) Jaffé, Ber. d. dtsch. chem. Gesellschft., Bd. 12, S. 1092.

6) Goldmann, Diese Zeitschr., Bd. IX, S. 260. 
Menschenhaaren und wurde im wesentlichen nach Mörners Vorschrift gewonnen. Verabreicht wurde das Cystin in wässeriger Suspension, da Lösungsmittel, wie Salzsäure, Ammoniak, Natronlauge, sich von selbst verboten. Eine abgewogene Menge des. Cystins wurde in der Reibschale mit Wasser fein verrieben und diese Suspension mittels Sonde und Spritze dem Tier unter Vermeidung von Luftzutritt in den Magen gespritzt; diese Methode der Darreichung wurde von sämtlichen Tieren (Kaninchen) gut vertragen. Der Versuch, dem Tier das Pulver unter die Nahrung gemischt beizubringen, mißglückte; es verweigerte tagelang die Nahrungsaufnahme.

Bevor ich aut die einzelnen Versuche näher eingehe, möchte ich, um mich nicht zu wiederholen, verschiedenes vorwegnehmen.

Was zunächst die Ernährung der Versuchstiere anbetrifft, so mußte man sich darüber klar sein, daß die einzelnen Futterstoffe verschieden auf die Ausscheidung der schwefelhaltigen Stoffwechselprodukte wirken, eine Tatsache, die durch Untersuchungen von Kunkel ') völlig klargelegt ist. Von diesem Gesichtspunkte ausgehend, hatte E. Salkowski) bei seinen Fütterungsversuchen mit Taurin die Kaninchen mit Kartoffeln ernährt, ein Material, das bekanntlich arm an Schwefel ist. Da jedoch die Tiere bei diesem Futter bisweilen die Freßlust verlieren, beschloß ich, die übliche Nahrung, bestehend in $300 \mathrm{~g}$ Mohrrüben und $300 \mathrm{~g}$ Kohl, anzuwenden. Außerdem hat diese Art der Ernährung den Vorteil, daß die Tiere dabei reichliche Mengen Urin produzieren, was für die zahlreichen Bestimmungen ein wichtiger Faktor ist.

Die Versuchsanordnung war folgende: ein Kaninchen wurde sechs Tage lang mit der oben beschriebenen Kost ernährt, und während dieser Zeit wurde der Urin in 2 tägigen Portionen auf seinen Gehalt an schwefelhaltigen Bestandteilen untersucht. Dann wurde dem Tier eine bestimmte Dosis Cystin verabreicht und der Urin 2-3 Tage hintereinander untersucht. Bestimmt wurde im Harn während der Beobachtungszeit der Gesamt-

1) Kunkel, Pflügers Archiv, Bd. 14, S. 344.

2) E. Salkowski, l. c. 
schwefel, die Gesamtschwefelsäure und die Ätherschwefelsäuren, und mittels Berechnung der neutrale Schwefel (Gesamtschwefel minus Gesamtschwefelsäure) und die Sulfate (Gesamtschwefelsäure minus Ätherschwefelsäure) festgestellt. Die Bestimmung des Gesamtschwefels wurde jedesmal mit $50 \mathrm{ccm}$ klar filtriertem Urin vorgenommen und unter genauer Beobachtung der Vorschriften in der Weise ausgeführt, wie sie E. Salkowski in seinem Praktikum angibt. Die Bestimmung der Schwefelsäure wurde an $100 \mathrm{ccm}$ Harn gemacht, der Urin mit Salzsäure gekocht, mit Chlorbaryum versetzt und die Fällung erst nach mindestens 12stündigemStehen abfiltriert etc. und gewogen. Da es anfänglich öfters vorkam, daß der Niederschlag sich nicht ganz klar absetzte, erhitzte ich jedesmal nach Zusatz von Chlorbaryum auf freiem Feuer oder auf dem Wasserbad und bekam dadurch stets klare Lösungen. Beim Kochen mit Salzsäure konnte ich, sobald ich den Urin eines mit Cystin gefütterten Tieres zu untersuchen hatte, stets einen heftig stechenden Geruch wahrnehmen und beobachtete in fast allen Fällen eine deutliche Trübung. Diese Trübung trat in der charakteristischen Weise auf, wie sie E. Salkowski bei seinen Taurinharnen beschreibt, der Harn trübte sich zuerst ganz gleichmäßig in der Art, daß er bei durchfallendem Licht noch klar und nur in auffallendem weißlich trübe erschien, dann nahm die Trübung allmählich zu, der Harn wurde ganz undurchsichtig, und endlich ballte sich der Schwefel in Flocken zusammen und setzte sich vollkommen ab. Von einer Wägung dieses Niederschlages wurde Abstand genommen, da diese Methode, wie schon E. Salkowski betont hat, keine genauen Resultate gibt. Ebensowenig ist die andere Methode der Bestimmung der unterschwefligen Säure - mittels Fällung mit Silberlösung und Behandeln des Niederschlages mit Ammoniak - frei von Fehlern und gibt gleichfalls $\mathrm{zu}$ hohe Werte. Zur genaueren Charakterisierung wurde der Niederschlag in siedendem Chloroform gelöst und die Lösung an der Luft langsam verdunstet. Es blieben dann glänzend weiße Krystallnädelchen zurück, die mit blauer Flamme ohne Rückstand und unter Entwicklung des typischen Geruchs nach schwefliger Säure verbrannten und subli- 
mierten. Damit war der Beweis erbracht, daß es sich um Schwefel handelt. In 3 Fällen schlug ich diesen Weg ein, in den übrigen begnügte ich mich damit, beim Kochen mit Salzsäure das Auftreten des stechenden Geruchs nach schwefliger Säure und die gleichförmige, allmählich flockig werdende Trübung zu konstatieren, was wohl beweisend für die Anwesenheit der unterschwefligen Säure sein dürfte, denn normaler Kaninchenharn weist niemals diese Eigenschaft auf. Ebenso zeigte der nach Cystinfütterung entleerte Harn das typische Verhalten des Thiosulfats gegen Silbernitrat, nur mit der Abweichung gegenüber der reinen Thiosulfatlösung, daß die Bildung von. Schwefelsilber in der Kälte sehr langsam erfolgte, schneller beim Erwärmen. Offenbar wirken die Harnbestandteile störend auf den Ablauf der Reaktion, wie ich durch Kontrollversuche feststellen konnte; und zwar scheinen die Chloride eine auf den Eintritt der Reaktion hemmende Wirkung auszuüben.

Die Menge des ausgeschiedenen neutralen Schwefels, die, wie ich oben schon sagte, durch Abzug der Schwefelsäure vom Gesamtschwefel berechnet wurde, hielt sich an den normalen Tagen in ziemlich bestimmten Grenzen, und zwar konnte ich ebenso wie E. Salkowski ${ }^{1}$ ) konstatieren, daß das Verhältnis des neutralen Schwefels zum oxydierten annähernd $1: 4$ ist. Zum Beleg stelle ich von sämtlichen 5 Versuchen die Bestimmungen der Normaltage zusammen:

Tabelle 1.

\begin{tabular}{c|c|c|c|c}
\hline $\begin{array}{c}\text { Nr. der } \\
\text { Versuchstiere }\end{array}$ & $\begin{array}{c}\mathrm{a} \\
\text { Gesamtschwefel }\end{array}$ & $\begin{array}{c}\mathrm{b} \\
\text { Gesamtschwefel- } \\
\text { säure }\end{array}$ & $\begin{array}{c}\mathrm{c} \\
\text { Neutraler } \\
\text { Schwefel }\end{array}$ & $\mathrm{c}: \mathrm{b}$ \\
\hline \multirow{3}{*}{ I } & 0,3475 & 0,2818 & 0,0657 & $1: 4,29$ \\
& 0,2739 & 0,2220 & 0,0519 & $1: 4,2$ \\
& 0,3089 & 0,2488 & 0,0601 & $1: 4,1$ \\
\hline \multirow{3}{*}{ II } & 0,3270 & 0,2468 & 0,0802 & $1: 3,07$ \\
& 0,3153 & 0,2482 & 0,0671 & $1: 3,7$ \\
& 0,3052 & 0,2321 & 0,0731 & $1: 3,1$ \\
\hline
\end{tabular}

1) E. Salkowski, l. c. 
Über die Herkunft der schwefelhaltigen Stoffwechselprodukte etc. 87

\begin{tabular}{c|c|c|c|c}
\hline \hline $\begin{array}{c}\text { Nr. der } \\
\text { Versuchstiere }\end{array}$ & $\begin{array}{c}\mathrm{a} \\
\text { Gesamtschwefel }\end{array}$ & $\begin{array}{c}\mathrm{b} \\
\text { Gesamtschwefel- } \\
\text { säure }\end{array}$ & $\begin{array}{c}\mathrm{c} \\
\text { Neutraler } \\
\text { Schwefel }\end{array}$ & $\mathbf{c}: \mathbf{b}$ \\
\hline \multirow{3}{*}{ III } & 0,6217 & 0,5031 & 0,1186 & $1: 4,2$ \\
& 0,4944 & 0,4089 & 0,0855 & $1: 4,7$ \\
& 0,5274 & 0,4175 & 0,1099 & $1: 3,4$ \\
\hline \multirow{3}{*}{ IV } & 0,5576 & 0,4453 & 0,1123 & $1: 3,96$ \\
& 0,5022 & 0,3907 & 0,1115 & $1: 3,5$ \\
& 0,5045 & 0,4148 & 0,0897 & $1: 4,6$ \\
\hline & 0,5744 & 0,4495 & 0,1249 & $1: 3,6$ \\
& 0,4686 & 0,3767 & 0,0919 & $1: 4,1$ \\
& 0,4886 & 0,4013 & 0,0873 & $1: 4,6$ \\
\hline
\end{tabular}

Dieses Verhältnis änderte sich ganz wesentlich nach Verfütterung von Cystin und zwar stets zugunsten des neutralen Schwefels, während der oxydierte Schwefel immer nur eine ganz geringe Steigerung erfuhr.

Um nun zu wissen, auf welchem Anteil der Schwefelsäure das Ansteigen derselben beruht - ob auf Zunahme der Sulfate oder der Ätherschwefelsäuren -, wurden jedesmal die Ätherschwefelsäuren bestimmt und die Menge der Sulfate durch Abzug der Ätherschwefelsäuren von der gesamten Schwefelsäure berechnet.

Ich gehe nunmehr auf die einzelnen Versuche selber über und möchte für alle Versuche bemerken, daß die in den Tabellen angeführten Zahlen sämtlich auf Sc hw ef el berechnet sind.

Versuch I.

Tabelle 2.

\begin{tabular}{|c|c|c|c|c|c|c|}
\hline Tage & $\begin{array}{c}\text { a } \\
\text { Gesamt- } \\
\text { schwefel }\end{array}$ & $\begin{array}{c}\text { b } \\
\text { Gesamt- } \\
\text { schwefel- } \\
\text { säure }\end{array}$ & $\begin{array}{c}c \\
\text { Neutraler } \\
\text { Schwefel }\end{array}$ & $\begin{array}{c}\mathrm{d} \\
\text { Äther- } \\
\text { schwefel- } \\
\text { säuren }\end{array}$ & $\begin{array}{c}\text { e } \\
\text { Sulfate }\end{array}$ & $c: b$ \\
\hline 1. u. 2. & 0,3475 & 0,2818 & 0,0657 & 0,0115 & 0,2703 & $1: 4,29$ \\
\hline 3. 4 . & 0,2739 & 0,2220 & 0,0519 & 0,0110 & 0,2110 & $1: 4,2$ \\
\hline $5 . \gg 6$. & 0,3089 & 0,2488 & 0,0601 & 0,0121 & 0,2367 & $1: 4,1$ \\
\hline 7. 8. & $\mathbf{0 , 5 8 6 3}$ & 0,3463 & 0,2400 & 0,0126 & 0,3337 & $1: 1,5$ \\
\hline $9 . » 10$. & 0,3439 & 0,2860 & 0,0579 & 0,0125 & 0,2735 & $1: 4,9$ \\
\hline 11. 12. & 0,6556 & 0,3795 & 0,2761 & 0,0118 & 0,3677 & $1: 2,1$ \\
\hline 13. 14. & 0,3293 & 0,2559 & 0,0734 & 0,0123 & 0,2436 & $1: 3,48$ \\
\hline
\end{tabular}


Kaninchen von $2070 \mathrm{~g}$ Gewicht erhält nach 6 tägiger Beobachtung am 7. Tage $3,0 \mathrm{~g}$ Cystin per os in wässeriger Suspension, dann nach wiederum 2 tägiger Pause am 11. Tag abermals $3,0 \mathrm{~g}$ Cystin in derselben Form.

\section{Urin}

vor der Fütterung

Reaktion: schwach alkalisch

Drehung: optisch inaktiv

Bleischwärzende kaum

Reaktion: $\}$ angedeutet nach der Fütterung schwach alkalisch optisch inaktiv schwach positiv.

Ein ähnliches oder fast gleiches Verhalten zeigten die verschiedenen Urinmengen in sämtlichen übrigen Versuchen, sodaß ich diesen Punkt später nicht nochmals zu berühren brauche.

Um einen Überblick zu bekommen von dem Verhältnis des ausgeschiedenen Schwefels zum eingegebenen, stellte ich folgende Berechnung an:

An den ersten 6 Tagen des Versuchs ist ausgeschieden a) Schwefelals Schwefelsäure 0,9326 mithin durchschnittlich 0,3109) für

b) Schwefel in andrer Form 0,1777 » $\quad 0,0592\} 2$ Tage

Am 7. u. 8. Tag ist nach Cystinfütterung ausgeschieden a) Schwefel als Schwefelsäure 0,3463 , mithin eine Steigerung gegen die Normaltage um $0,3463-0,3109=0,0354 \mathrm{~g}$

b) Schwefel in andrer Form 0,2400, mithin eine Steigerung

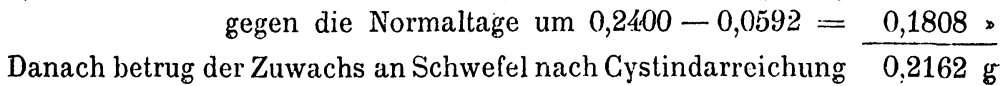

Dieses Plus von 0,2162 g Schwefel kann man somit als vom Gystin herrührend anselen. Auf Cystin berechnet würden folglich $0,82 \mathrm{~g}$ Cystin den Körper passiert haben, ditvon wären $16,4 \%$ als Schwefelsäure und $83,6 \%$ als neutraler Schwefel wieder im Harn erschienen. Cystin als solches war im Harn nicht vorhanden, was schon aus der optischen Inaktivität, dem fast negativen Ausfall der bleischwärzenden Reaktion und aus. dem. Fehlen jeglichen Sediments deutlich hervorging. Am 9. und 10. Tag war die Schwefelausfuhr wieder auf die Norm zurückgegangen, um an den beiden nächsten Tagen nach Darreichung von $3,5 \mathrm{~g}$ Cystin per os wiederum erheblich anzusteigen. Es wurden am 11. und 12. Tag nach Cystinfütterung ausgeschieden 
a) Schwefel als Schwefelsäure 0,3795, mithin eine Steigerung

gegen die Normaltage um $0,3795-0,3109=$
b) Schwefel in andrer Form 0,2761, davon ab $0,0592=\frac{0,0686 \mathrm{~g}}{0,2169}$

Das Plus von 0,2855 g Schwefel wäre nach der ersten Fütterung auf Cystin zu beziehen und würde 1,07 g Cystin entsprechen. Hiervon sind somit $24,8 \%$ als Schwefelsäure und $75,2 \%$ als neutraler Schwefel ausgeschieden. Schon bei der Schwefelsäurebestimmung nach der ersten Cystinfütterung war mir ein stechender Geruch aufgefallen, während gleichzeitigeine deutliche Trübung auftrat. Indes erst nach der 2. Fütterung war der Niederschlag im Urin nach Kochen mit HCl so reichlich, daß es sich lohnte, ihn abzufiltrieren und mit heißem Chloroform in der bereits oben beschriebenen Weise zu behandeln; es konnte einwandfrei nachgewiesen werden, daß der Niederschlag zum größten Teil aus Schwefel bestand.

Es fragt sich nun, wo das übrige Cystin geblieben ist. Da nur ein Bruchteil des Schwefels im Harn wieder erschienen ist, lag es zunächst nahe, anzunehmen, daß das Cystin nur zum Teil resorbiert, zum Teil wieder durch den Darm ausgeschieden worden ist. Es war ja von vornherein klar, daß die Art, wie den Tieren das Cystin gegeben wurde, für die Resorption nicht günstig war. Denn es ist immer mißlich, einem Kaninchen viel Wasser in den Magen zu bringen, da die Tiere sehr leicht Durchfall bekommen.

Um die Frage von dem Verbleib des übrigen Cystins zu lösen, wurden die Faeces von dem Cystin- und dem darauffolgenden Tage mit verdünntem Ammoniak extrahiert, der tiefdunkle Auszug zur Entfärbung mit Knochenkohle gekocht und dann auf Bleireaktion und Polarisation untersucht. Beide Reaktionen fielen negativ aus. Man konnte nun annehmen, daß die Substanz im Magen des Kaninchens längere Zeit zurückgehalten und erst nach einigen Tagen zur Ausscheidung gelangen würde; aber auch die Extraktion der Faeces der folgenden Tage mittels verdünnten Ammoniaks war ergebnislos.

\section{Versuch II.}

Kaninchen von $1930 \mathrm{~g}$ Gewicht erhält nach 6tägiger Beobachtung 5,0 g Cystin per os in wässeriger Suspension. 
Tabelle 3 .

\begin{tabular}{|c|c|c|c|c|c|c|}
\hline Tage & $\begin{array}{c}\text { a } \\
\text { Gesamt- } \\
\text { schwefel }\end{array}$ & $\begin{array}{c}\mathrm{b} \\
\text { Gesamt- } \\
\text { schwefel- } \\
\text { säure }\end{array}$ & $\begin{array}{c}\text { c } \\
\text { Neutraler } \\
\text { Schwefel }\end{array}$ & $\begin{array}{c}\mathrm{d} \\
\text { Äther- } \\
\text { schwefel- } \\
\text { säuren } \\
\end{array}$ & $\begin{array}{c}\text { e } \\
\text { Sulfate }\end{array}$ & $c: b$ \\
\hline 1. u. 2. & 0,3270 & 0,2468 & 0,0802 & 0,0107 & 0,2361 & $1: 3,07$ \\
\hline 3. $\searrow$. & 0,3153 & 0,2482 & 0,0671 & 0,0097 & 0,2412 & $1: 3,7$ \\
\hline 5. $\gg 6$. & 0,3052 & 0,2321 & 0,07331 & 0,0120 & 0,2201 & $1: 3,1$ \\
\hline 7. 8. & 0,6922 & 0,4198 & 0,2724 & 0,0123 & 0,4075 & $1: 1,2$ \\
\hline $9 . » 10$. & 0,4110 & 0,2858 & 0,1252 & 0,0121 & 0,2737 & $1: 2,2$ \\
\hline 11. 12. & 0,3233 & 0,2388 & 0,0845 & 0,0125 & 0,2263 & $1: 2,8$ \\
\hline
\end{tabular}

Ausgeschieden ist in den ersten 6 Tagen des Versuchs an Schwefel

a) als Schwefelsäure 0,7271, mithin durchschnittlich 0,2424 \} für

b) in andrer Form 0,2204, " $, 0,0735$ \} 2 Tage

Am 7. und 8. Tag nach der Cystinfütterung sind ausgeschieden an Schwefel

a) als Schwefelsäure 0,4198, davon gehen ab $0,2424=0,1774 \mathrm{~g}$

b) in andrer Form 0,2724, » $\gg 0,0735=\frac{0,1989 \text {, }}{0,3763 \mathrm{~g}}$

$0,3763 \mathrm{~g}$ Schwefel sind somit auf das Cystin zu beziehen. Auf Cystin berechnet würden folglich 1,43 g Cystin zur Resorption gekommen sein; davon wären $47,1 \%$ als Schwefelsäure und $52,9 \%$ als neutraler Schwefel wieder im Harn erschienen. Das Ansteigen der Schwefelsäureausfuhr beruht, wie aus der Tabelle ersichtlich, auf der Zunahme der Sulfate; die Menge der ausgeschiedenen Ätherschwefelsäuren blieb in den Grenzen der vorhergehenden T'age.

Am 9. und 10. Tag ist noch eine merkliche Steigerung der Schwefelausfuhr zu verzeichnen. Es wurde ausgeschieden an Schwefel

a) als Schwefelsäure 0,2858, davon gehen ab $0,2424=0,0434 \mathrm{~g}$

b) in andrer Form 0,1252, $>0,0735=\frac{0,0517 \text { » }}{0,0951 \mathrm{~g}}$

Dieses Plus von $0,0951 \mathrm{~g}$ Schwefel wäre ebenfalls auf Cystin $\mathrm{zu}$ beziehen und würde etwa $0,41 \mathrm{~g}$ Cystin entsprechen.

An den beiden nächstfolgenden Tagen hatte sich die Schwefelausfuhr wieder der Norm fast genähert. Es hatten somit von 5,0 g Cystin im ganzen 1,84 g den Blutkreislauf 
Über die Herkunft der schwefelhaltigen Stoffwechselprodukte etc. 91

passiert. Um über den Verbleib der übrigen Cystinmenge ein Urteil zu haben, wurden auch diesmal die Faeces der letzten 6 Tage in der schon angegebenen Weise verarbeitet, indes auch hier mit negativem Resultat.

Natürlich befriedigte dieses Ergebnis nicht und es war nun daran zu denken, daß der Tierkörper, überschwemmt mit so großen Cystinmassen, das Cystin als solches in seinen Organen deponiert hat, ähnlich wie es in Fällen von Cystinurie neuerdings konstatiert wurde. Dann aber war auch $\mathrm{zu}$ erwägen, ob das Cystin nicht - teilweise abgebaut - in einem solch veränderten Zustande im Körper retiniert worden war.

Um hierin Klarheit zu bekommen, war es in erster Linie notwendig, den Schwefelgehalt der Organe normaler Tiere zu bestimmen. $\mathrm{Zu}$ dem Zwecke tötete ich 3 Kaninchen und verarbeitete deren Galle, Leber und je $100 \mathrm{~g}$ Muskelsubstanz. Andere Organe in den Kreis der Untersuchung zu ziehen, schien mir überflüssig. In der Galle wurde der Schwefelgehalt nach dem Vorgange von Gorup-Besanez in der Weise bestimmt, daß die Galle mit Alkohol extrahiert, der alkoholische Auszug - nach Trennung der Fällung durch Abfiltrieren - in einer Platinschale verdampft und dann mit Salpeter und Soda geschmolzen wurde. Die Schmelze wurde in Wasser gelöst, dreimal mit Salzsäure abgedampft, um sämtliche Salpetersäure zu vertreiben, filtriert und mit Chlorbaryum versetzt. Der Niederschlag von schwefelsaurem Baryt wurde nach mindestens zwölfstündigem Stehen abfiltriert, gewogen, auf Schwefel umgerechnet und daraus der Prozentgehalt an Schwefel im alkoholischen Auszug der Galle bestimmt. Die Feststellung des Schwefelgehalts in der Leber war etwas umständlicher. Von der Erwägung ausgehend, daß das möglicherweise zurückgehaltene Cystin entweder als solches oder als ein Derivat desselben in der Leber sich vorfinden konnte, mußte ich zunächst darauf bedacht sein, diesen unbekannten Körper von der Lebersub$\operatorname{stanz}$ zu trennen. Zu dem Zwecke wurde die Leber fein gehackt, gewogen und mit $200 \mathrm{ccm}$ Wasser auf freiem Feuer kurze Zeit gekocht. Da man aber beim Kochen der Leber mit Wasser stets einen Teil des an sich schon schwefelhaltigen 
Nucleoproteids ${ }^{1}$ ) in Lösung bekommt, wurde der Auszug nach dem Erkalten mit Essigsäure versetzt und von den abgeschiedenen Proteiden abfiltriert. Darauf wurde die Lösung in einer Platinschale eingeengt und dann in derselben Weise weiter verarbeitet wie der alkoholische Auszug der Galle. Ein wässeriger Auszug von der Leber genügte jedoch nicht, um einen vollkommenen Überblick über sämtliche freien schwefelhaltigen Bestandteile in der Leber zu gewähren. War z. B. Cystin in der Leber vorhanden, so braucht dieses nicht in den wässerigen Auszug übergehen. Aus diesem Grunde wurden, um für die späteren Versuche vergleichende Resultate zu haben, auch die Lebern der normalen Tiere nach ihrer Extraktion mit Wasser noch mit verdünnter Salzsäure extrahiert, und zwar wurde der Leberrückstand mit $200 \mathrm{ccm} 5^{0}{ }^{\circ}$ iger $\mathrm{HCl} 2-3$ Minuten lang gekocht, die Salzsäurelösung abfiltriert und der Rückstand auf dem Filter mehrmals mit heißem Wasser gewaschen. In der gesamten Lösung wurde dann der Schwefelgehalt in derselben Weise bestimmt wie beim wässerigen Extrakt. Von der Muskelsubstanz - es wurden jedem Kaninchen $100 \mathrm{~g}$ Muskel entnommen - wurde ebenfalls sowohl ein wässeriger wie salzsaurer Auszug hergestellt und mit diesen Extrakten die Schwefelbestimmung in der üblichen Art vorgenommen. Sämtliche Auszüge nun - die der Galle, der Leber und der Muskel - gaben niemals oder nur ganz schwache positive bleischwärzende Reaktion.

Die so gefundenen Werte, die sich, wie schon gesagt, alle auf Schwefel beziehen, sind in folgender Tabelle zusammengestellt.

Tabelle 4. (Normale Kaninchen.)

\begin{tabular}{c|c|c|c|c|c}
\hline \hline \multirow{2}{*}{$\begin{array}{c}\text { Vor- } \\
\text { versuchstier }\end{array}$} & \multirow{2}{*}{ Galle } & \multicolumn{2}{|c|}{ Leberauszug } & \multicolumn{2}{|c}{ Muskelauszug } \\
& & wässerig & sauer & wässerig & sauer \\
\hline 1. & $2,28 \%$ & $2,08 \%$ & $0,66 \%$ & $0,48 \%$ & $0,78 \%$ \\
2. & $1,5 \%$ & $2,08 \%$ & $0,76 \%$ & $0,91 \%$ & $0,97 \%$ \\
3. & $2,3 \%$ & $2,07 \%$ & $1,02 \%$ & $0,52 \%$ & $0,73 \%$ \\
& & & &
\end{tabular}

1) J. Wohlgemuth, Diese Zeitschrift, Bd. XXXVII, S. 475. 1903. 
Über die Herkunft der schwefelhaltigen Stoffwechselprodukte etc. 93

Demnach enthält durchschnittlich an Schwefel: die Galle eines Kaninchens $2,03 \%$ der wässerige Auszug der Leber $2,08 \%$ der saure $\quad$ " $0,81 \%$ der wässerige Auszug der Muskeln 0,64\% der saure $\quad$ » $0,83 \%$

Nach diesen orientierenden Bestimmungen ging ich abermals daran, Fütterungsversuche an Kaninchen mit Cystin vorzunehmen. Ich stellte diese Versuche in genau der gleichen Weise an, wie die beiden ersten: das Versuchstier erhielt das übliche Futter, wurde sechs Tage beobachtet, während dieser Zeit der Urin auf seine schwefelhaltigen Stoffwechselprodukte untersucht und bekam am 7. Tage ein bestimmtes Quantum Gystin. Nach 36-48 Stunden wurde es getötet und der Urin und die Organe in der oben beschriebenen Weise verarbeitet.

Versuch VI.

Tabelle $\check{5}$.

\begin{tabular}{|c|c|c|c|c|c|c|}
\hline Tage & $\begin{array}{c}\text { a } \\
\text { Gesamt- } \\
\text { schwefel }\end{array}$ & $\begin{array}{c}\text { b } \\
\text { Gesamt- } \\
\text { schwefel- } \\
\text { säure }\end{array}$ & $\begin{array}{c}\text { c } \\
\text { Neutraler } \\
\text { Schwefel }\end{array}$ & $\begin{array}{c}\mathrm{d} \\
\text { Äther- } \\
\text { schwefel- } \\
\text { säuren }\end{array}$ & $\begin{array}{c}\text { e } \\
\text { Sulfate }\end{array}$ & $c: b$ \\
\hline 1. u. 2. & 0,6217 & 0,5031 & 0,1186 & 0,0309 & 0,4722 & $1: 4,2$ \\
\hline 3. $\$ 4$ & 0,4944 & 0,4089 & 0,0855 & 0,0313 & 0,3776 & $1: 4,7$ \\
\hline $5 . \gg 6$. & 0,5274 & 0,4175 & 0,1099 & 0,0344 & 0,3831 & $1: 3,4$ \\
\hline 7. 8 . & 0,7302 & 0,4638 & 0,2664 & 0,0340 & 0,4298 & $1: 1,7$ \\
\hline
\end{tabular}

Kaninchen von $1870 \mathrm{~g}$ Gewicht erhält am 7. Tage $3 \mathrm{~g}$ Gystin per os in wässeriger Suspension; nach 48 Stunden wird es getötet.

Der Urin zeigte vor und nach der Fütterung keine von den in Betracht kommenden Reaktionen.

Was die Schwefelausfuhr anbetrift, so ist während der ersten sechs Tage ausgeschieden an Schwefel:

a) als Schwefelsäure 1,3295, mithin durchschnittlich 0,4432 für je

b) in anderer Form 0,3140 , $, 0,1047$ \} 2 Tage

Am 7. und 8. Tage nach der Cystinfütterung wurde Schwefel ausgeschieden: 
a) als Schwefelsäure 0,4638 davon abzüglich $0,4432=0,0206 \mathrm{~g}$

b) in anderer Form 0,2664 » $\gg, 1047=\frac{0,1617 \text { » }}{0,1823 \mathrm{~g}}$

$0,1823 \mathrm{~g}$ Schwefel kann man mithin als vom Cystin herrührend ansehen. Auf Cystin berechnet, würden folglich $0,69 \mathrm{~g}$ Cystin den Blutkreislauf passiert haben; davon wären $21,3 \%$ als Schwefelsäure und $87,7 \%$ als neutraler Schwefel im Harn wieder erschienen.

Bei der Bestimmung der Schwefelsäure im Harn (Erhitzen mit $\mathrm{HCl}$ ) traten auch dieses Mal der typisch stechende Geruch und die anfangs undeutliche, später immer stärker werdende Trübung auf, bis sich schließlich die weißen Partikelchen zu größeren Flocken zusammenballten - ein Beweis, daß auch in diesem Harn unterschwefelige Säure enthalten war. Die Reindarstellung des Schwefels unterblieb bei diesem Versuch.

Von dem getöteten Tier wurden Galle, Leber und Muskeln in der oben beschriebenen Weise auf ihren Schwefelgehalt verarbeitet. Sowohl die Leber wie die Muskelsubstanz und alle anderen Organe zeigten nirgends makroskopische Ablagerungen von Cystin; ebenso konnte ich in den beiden folgenden Versuchen niemals eine derartige Beobachtung machen.

Die Galle hatte einen Gehalt an Schwefel von 6,68\% gegen 2,03\%; der wässerige Auszug der Leber " $\quad$ " $\mathbf{5 , 8 2} \%$ " $2,08 \%$; der saure $>>, \quad, \mathbf{2 , 0 2} \%$ " $0,81 \%$; der wässerige Auszug der Muskeln » $\quad \triangleright \quad 0,81 \%$, $0,64 \%$; der saure " $\gg>\gg, 1,05 \%$

Danach haben einen beträchtlichen Zuwachs an Schwefel erfahren: die Galle, und zwar um mehr als das Dreifache und der wässerige Leberauszug um mehr als das $2^{1} / 2$-fache. Eine geringe Zunahme zeigten die übrigen Auszüge. Was speziell die salzsauren Auszüge der Leber und Muskeln anbetrifft, so war der Schwefelgehalt an sich nicht unbeträchtlich. Indes war derselbe nicht auf Cystin zu beziehen, da die bleischwärzende Reaktion mit dem Auszug negativ ausfiel. Vielmehr konnte man vermuten, daß beim Kochen mit Salzsäure Eiweiß in Lösung gegangen war und der in diesem Eiweiß enthaltene 
Schwefel bei der Schwefelbestimmung eine wesentliche Rolle spielt. Aus diesem Grunde wurde die andere noch nicht verarbeitete Hälfte des Extraktes mit $\mathrm{NaOH}+\mathrm{NaCl}$ versetzt, wobei ein starker Ausfall von Acidalbumin auftrat. Mit der so eiweißfrei gemachten Lösung nun wurde die Schwefelbestimmung ausgeführt; die Niederschläge nach Zusatz von Chlorbaryum waren jedoch sowohl in dem sauren Leber- wie sauren Muskelextrakt so gering, daß sie kaum gewogen werden konnten. Die Werte für diese beiden Auszüge wurden deshalb auch in den späteren Versuchen nicht weiter berücksichtigt, ich habe sie aber der Vollständigkeit halber stets angeführt.

Die Summe des im Harn und in den Organen wieder erschienenen Schwefels entsprach aber auch in diesem Falle keineswegs der eingegebenen Cystinmenge. Ich untersuchte deshalb den ganzen Darminhalt des getöteten Tieres auf Cystin, indem ich denselben dieses Mal mit starker Ammoniaklösung lange erhitzte. In einem Teil dieser Lösung wurde der Schwefel in der üblichen Weise bestimmt und ergab den Wert von 0,4397 g Schwefel. Die andere Hälfte der Lösung wurde auf dem Wasserbade eingeengt und war nach längerem Stehen zu einem dicken Brei erstarrt. Durch Absaugen wurde die Substanz von der Mutterlauge getrennt, dann aus Ammoniak umkrystallisiert, auf einem gewogenen Gooch-Tiegel gesammelt, getrocknet und gewogen. Ihr Gewicht betrug 1,5037 g. Die Substanz konnte durch ihr typisches mikroskopisches Bild (kleine sechseckige regelmäßige Täfelchen) und durch den positiven Ausfall der bleischwärzenden Reaktion schon in ganz geringen Mengen als reines Cystin charakterisiert werden. Mit diesem Befund war auch gleichzeitig Klarheit geschaffen über den Weg, den das verfütterte Cystin im Körper des Kaninchens nimmt. Danach ist man berechtigt, anzunehmen, daß der größte Teil des Cystins unverändert durch den Darm wieder ausgeschieden, der kleinere Teil zur Resorption kommt, die Leber passiert und von da in die Galle wandert oder in den Blutkreislauf übergeht. In welcher Form es sich in der Leber und in der Galle vorfindet, das soll später erörtert werden. 
Versuch VII.

Tabelle 6 .

\begin{tabular}{|c|c|c|c|c|c|c|}
\hline Tage & $\begin{array}{c}\text { a } \\
\text { Gesamt- } \\
\text { schwefel }\end{array}$ & $\begin{array}{c}\text { b } \\
\text { Gesamt- } \\
\text { schwefel- } \\
\text { säure }\end{array}$ & $\begin{array}{c}c \\
\text { Neutraler } \\
\text { Schwefel }\end{array}$ & $\begin{array}{c}\mathrm{d} \\
\text { Äther- } \\
\text { schwefel- } \\
\text { säuren }\end{array}$ & $\begin{array}{c}\text { e } \\
\text { Sulfate }\end{array}$ & $c: b$ \\
\hline 1. u. 2. & 0,5576 & 0,4453 & 0,1123 & 0,0276 & 0,4177 & $1: 3,96$ \\
\hline 3. 4 . & 0,5022 & 0,3907 & 0,1115 & 0,0368 & 0,3อ̆39 & $1: 3,5$ \\
\hline $5 .>6$. & 0,5045 & 0,4148 & 0,0897 & 0,0355 & 0,3813 & $1: 4,6$ \\
\hline 7. 8. & 0,7219 & 0,4641 & 0,2578 & 0,0255 & 0,4386 & $1: 1,8$ \\
\hline
\end{tabular}

Kaninchen von $2150 \mathrm{~g}$ Gewicht erhält nach 6 tägiger Beobachtung 3,5 g Cystin per os. Nach 36 Stunden wird es getötet.

Es hat in den ersten 6 Tagen des Versuchs ausgeschieden an Schwefel:

a) als Schwefelsäure 1.2508 , mithin durchschnittlich $0,4169 \mathrm{~g}$

b) als neutraler Schwefel 0,3135, » $~ 0,1012$,

In den letzten 36 Stunden nach der Cystinfütterung sind ausgeschieden an Schwefel:

a) als Schwefelsäure 0,4641 , davon gehen ab $0,4169=0,0472 \mathrm{~g}$

b) als neutraler Schwefel 0,2578, » » $0,1012=\frac{0,1566 \text { * }}{0,20: 38 \mathrm{~g}}$

0,2038 g Schwefel kann man also als vom Cystin stammend betrachten. Auf Cystin berechnet, würden somit $0,77 \mathrm{~g}$ den Blutkreislauf passiert haben, davon wären $23,1^{\circ} \%$ als Schwefelsäure und 76,9\% als neutraler Schwefel wieder im Harn erschienen. Beim Kochen des Harns mit Salzsäure trat auch in diesem Falle der typisch stechende Geruch und der Niederschlag auf. Derselbe wurde mit heißem Chloroform behandelt und aus ihm der Schwefel rein dargestellt. Die weißen spießigen Nädelchen verbrannten mit blauer Flamme, entwickelten dabei den charakteristischen Geruch nach schwefliger Säure und sublimierten. Somit enthielt auch dieser Harn unterschweflige Säure. Was nun die Organe des getöteten Tieres anbetrifft, so zeigten auch diese makroskopisch keine Veränderungen. Ihr Schwefelgehalt wurde in der oben beschriebenen Weise bestimmt und ergab: 
Über die Herkunft der schwefelhaltigen Stoffwechselprodukte etc. 97

1. für die Galle einen Gehalt von $\mathbf{5 , 0 5} \%$ gegenüber $2,03 \%$

2. für den wässerigen Leberauszug » $\gg, 6,02 \%$, $2,08 \%$

3. für den sauren $>\gg, \mathbf{2 , 0} \%$ "

4. für den wässerigen Muskelauszug » » $\gg \mathbf{0 , 8 4} \%$ \ $0,64 \%$

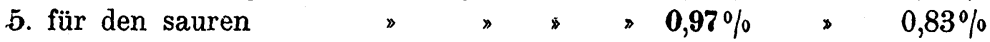

Wir sehen also auch in diesem Versuch ein beträchtliches Ansteigen des Schwefelgehalts der Galle und zwar um das $21 / 2$ fache über den Durchschnitt und ebenso eine erhebliche Zunahme an Schwefel im wässerigen Auszug der Leber. Wesentlich geringer ist die Steigerung im sauren Auszug der Leber und ganz unbedeutend in beiden Muskelextrakten. Da im vorigen Versuch Cystin in den Faeces in großer Menge nachgewiesen wurde, wurde hier auf die Untersuchung des Darminhaltes verzichtet und angenommen, daß auch dieses Mal ein Teil des verabfolgten Cystins nicht resorbiert worden war.

Versuch VIII.

Tabelle 7 .

\begin{tabular}{l|c|c|c|c|c|c}
\hline \hline Tage & $\begin{array}{c}\mathrm{a} \\
\text { Gesamt- } \\
\text { schwefel }\end{array}$ & $\begin{array}{c}\mathrm{b} \\
\text { Gesamt- } \\
\text { schwrefel- } \\
\text { säure }\end{array}$ & $\begin{array}{c}\mathrm{c} \\
\text { Neutraler } \\
\text { Schwefel }\end{array}$ & $\begin{array}{c}\mathrm{d} \\
\text { Äther- } \\
\text { schwefel- } \\
\text { säuren }\end{array}$ & $\begin{array}{c}\mathrm{e} \\
\text { Sulfate }\end{array}$ & $\mathbf{c : \mathbf { b }}$ \\
\hline 1. u. 2. & 0,5744 & 0,4495 & 0,1249 & 0,0147 & 0,43348 & $1: 3,6$ \\
3.»4. & $\mathbf{0 , 4 6 8 6}$ & 0,3767 & 0,0913 & 0,0149 & 0,3618 & $1: 4,1$ \\
5. 》6. & 0,4886 & 0,4013 & 0,0873 & 0,0086 & 0,3927 & $1: 4,6$ \\
7. 8.8. & $\mathbf{0 , 7 9 9 2}$ & $\mathbf{0 , 4 7 9 5}$ & $\mathbf{0 , 3 1 9 7}$ & $\mathbf{0 , 0 1 3 1}$ & $\mathbf{0 , 4 6 6 4}$ & $\mathbf{1 : 1 , 5}$
\end{tabular}

Kaninchen von $2580 \mathrm{~g}$ Gewicht wird 6 Tage hindurch bei der üblichen Kost heobachtet und bekommt am 7. Tage $3 \mathrm{~g}$ Cystin per os. Nach 48 Stunden wird es getötet.

An den ersten 6 Tagen des Versuchs sind ausgeschieden an Schwefel:

a) als Schwefelsäure $\quad 1,2275$, mithin durchschnittlich $0,4092 \mathrm{~g}$

b) als neutraler Schwefel 0,3035, » , 0,1012,

Am 7. und 8. Tag nach der Cystinfütterung wurde ausgeschieden an Schwefel;

a) als Schwefelsäure 0,4795 , davon gehen ab $0,4092=0,0703 \mathrm{~g}$

b) als neutraler Schwefel 0,3197, » $>0,1012 \frac{=0,2185 \text {, }}{0,2888 \mathrm{~g}}$ 
Danach haben 0,2888 g Schwefel $=1,09 \mathrm{~g}$ Cystin den Kreislauf passiert, und zwar sind $24,3 \%$ als Schwefelsäure und 75,7\% als neutraler Schwefel im Harn wieder erschienen.

Auch dieser Harn enthielt unterschweflige Säure; beim Kochen mit Salzsäure trat neben dem stechenden Geruch die Trübung resp. Fällung auf. Der Niederschlag wurde mit Chloroform behandelt und konnte als Schwefel charakterisiert werden.

Die Organe des getöteten Tieres zeigten folgenden Gehalt. an Schwefel:

1. die Galle

einen solchen von $\mathbf{7 , 3 4} \%$ gegenüber $2,03 \%$.

2. der wässerige Leberauszug » $\gg \quad, \mathbf{5}, \mathbf{0 6} \%$ » $2,08 \%$.

3. der saure $\gg>>1,90 \%$ "

4. der wässerige Muskelauszug 》 $~ \gg, 0,94 \%$ " $\%, 64 \%$

5. der saure $>>0,99 \%$ " $0,83 \%$

Auch dieses Mal beobachteten wir ein beträchtliches Ansteigen des Schwefels in der Galle um mehr als das $3^{1 / 2}$ fache und eine beträchtliche Steigerung des Schwefels im wässerigen Leberauszug und zwar um mehr als das $21 / 2$ fache. Die übrigen gefundenen Werte sind ohne Belang und können vernachlässigt werden.

\section{Ergebnisse.}

Aus den beiden ersten und den drei letzten Versuchen geht, was zunächst das Verhalten des Harns anbetrifft, hervor, daß das einem Kaninchen verabreichte Cystin eine Vermehrung der Schwefelsäure und zwar der Sulfate und eine erhebliche Steigerung des Gehalts an nicht oxydiertem Schwefel hervorruft. Diese Zunahme des neutralen Schwefels überwiegt derart den oxydierten Schwefel, daß das Verhältnis der beiden zu einander sich wesentlich verschiebt und von 1:4 wie in den normalen Tagen bis auf $1: 1,2$ (Versuch II) herabsinkt.

Mit dieser vermehrten Ausfuhr von neutralem Schwefel geht stets eine Ausscheidung von unterschwefligsauren Salzen einher. Regelmäßig zeigte der Harn beim Erhitzen mit Salzsäure die in ihrem Verlauf so charakteristische Trübung, 
die auf die Ausscheidung von Schwefel beruht, und den stechenden Geruch nach schwefliger Säure.

Bei der naheliegenden Möglichkeit der Umwandlung von Cystin in Taurin konnte kaum daran gezweifelt werden, daß das Cystin in der Tat im Organismus in Taurin übergegangen war. Den endgültigen Beweis hierfür liefert die Untersuchung der Galle und der Leber. Der Schwefelgehalt des alkoholischen Auszugs der Galle sowohl als auch der des wässerigen Leberextraktes zeigten sich gegen die Norm erheblich gesteigert, wie aus der folgenden Tabelle, in der ich die erhaltenen Werte noch einmal übersichtlich zusammengestellt habe, deutlich hervorgeht.

Tabelle 8.

\begin{tabular}{c|c|c|c|c|c}
\hline \multirow{2}{*}{$\begin{array}{c}\text { Nr. des } \\
\text { Versuchs }\end{array}$} & \multirow{2}{*}{ Galle } & \multicolumn{2}{|c|}{ Leberauszug } & \multicolumn{2}{|c}{ Muskelauszug } \\
& & wässerig & sauer & wässerig & sauer \\
\hline $\begin{array}{c}\text { Vorversuche } \\
\text { Durch- }\end{array}$ & \multirow{2}{*}{$2,03 \%$} & $2,08 \%$ & $0,81 \%$ & $0,64 \%$ & $0,83 \%$ \\
schnittszahl & & & & \\
6. & $\mathbf{6 , 6 8} \%$ & $\mathbf{5 , 8 2} \%$ & $\mathbf{2 , 0 2} \%$ & $\mathbf{0 , 8 1} \%$ & $\mathbf{1 , 0 5} \%$ \\
7. & $\mathbf{5 , 0 5} \%$ & $\mathbf{6 , 0 2} \%$ & $\mathbf{9 , 0} \%$ & $\mathbf{0 , 8 4} \%$ & $\mathbf{0 , 9 7} \%$ \\
8. & $\mathbf{7 , 3 4} \%$ & $\mathbf{5 , 0 6} \%$ & $\mathbf{1 , 9 0} \%$ & $\mathbf{0 , 9 4} \%$ & $\mathbf{0 , 9 9} \%$
\end{tabular}

Damit ist bewiesen, daß per os verabreichtes Cystin, soweit es resorbiert wird, in Taurin übergeht und zum Teil wenigstens als Taurocholsäure in der Galle erscheint.

Da das Cystin normalerweise bei der Pankreasverdauung des Eiweißes entsteht, so ist damit die Frage nach der Entstehung des Taurins im Organismus gelöst. $\mathrm{Zu}$ untersuchen bleibt noch, ob die Abspaltung von Cystin auf die Trypsinverdauung beschränkt ist, oder ob dasselbe, was wahrscheinlich ist, auch durch die Gewebsfermente bei der Autolyse entsteht.

Diese Untersuchungen waren Ende Juli abgeschlossen; der Beginn der Sommerferien verhinderte die Drucklegung. Inzwischen ist eine Arbeit von v. Bergmann ${ }^{1}$ ) über denselben Gegenstand erschienen. Beim Vergleich seiner Resultate mit

1) v. Bergmann, Hofmeisters Beiträge. Bd. 3, 1903. 
100 J. Wohlgemuth. Herkunft d. schwefelhalt. Stoffwechselprodukte.

den meinen ergibt sich, daß beide Tierspecies sich verschieden verhalten, indem beim Hunde die Zufuhr von Cystin nicht ohne weiteres eine Anreicherung des Schwefels in der Galle zur Folge hat, sondern nur bei gleichzeitiger Zufuhr von cholsaurem Natron, welches das entstehende Taurin als Taurocholsäure zu binden vermag. Dagegen möchte ich betonen, daß beim Kaninchen nach Cystinfütterung eine direkte Schwefelanreicherung der Galle stattfindet ohne jegliche Zufuhr einer schwefelbindenden Substanz. Möglicherweise bildet aber auch der Hund ohne Zuführung von Cholsäure Taurin und scheidet dasselbe entweder unverändert oder als Taurocarbaminsäure im Harn aus. Diese Frage könnte nur durch eine gleichzeitige eingehende Untersuchung des Harns entschieden werden. 